\title{
TRIPLE JUNCTION SOLUTIONS FOR A SINGULARLY PERTURBED NEUMANN PROBLEM
}

\author{
WEIWEI AO, MONICA MUSSO, AND JUNCHENG WEI
}

\begin{abstract}
We consider the following singularly perturbed Neumann problem

$$
\varepsilon^{2} \Delta u-u+u^{p}=0, \quad u>0 \quad \text { in } \Omega, \quad \frac{\partial u}{\partial \nu}=0 \quad \text { on } \quad \partial \Omega,
$$

where $p>1$ and $\Omega$ is a smooth and bounded domain in $\mathbb{R}^{2}$. We construct a class of solutions which consist of large number of spikes concentrating on three line segments with a common endpoint which intersect $\partial \Omega$ orthogonally .
\end{abstract}

\section{InTRODUCTION AND STATEMENT OF MAIN RESULTS}

We consider the following singularly perturbed elliptic problem

$$
\varepsilon^{2} \Delta u-u+u^{p}=0, \quad u>0 \quad \text { in } \Omega, \quad \frac{\partial u}{\partial \nu}=0 \quad \text { on } \partial \Omega,
$$

where $\Omega$ is a smooth bounded domain in $\mathbb{R}^{2}$ with its unit outer normal $\nu$, $p>1$ and $\varepsilon>0$ is a small parameter.

Problem (1.1) is known as the stationary equation of the Keller-Segel system in chemotaxes [25]. It can also be viewed as a limiting stationary equation for the Geirer-Meinhardt system in biological pattern formation [16]. Even though simple-looking, problem (1.1) has a rich and interesting structure of solutions. For the last fifteen years, it has received considerable attention. In particular, the various concentration phenomena exhibited by the solutions of (1.1) seem both mathematically intriguing and scientifically useful. We refer to three survey articles [35], [36] and [44] for backgrounds and references.

In the pioneering papers $[37,38], \mathrm{Ni}$ and Takagi proved the existence of least energy solutions to (1.1), that is, a solution $u_{\epsilon}$ with minimal energy. Furthermore, they showed in $[37,38]$ that, for each $\epsilon>0$ sufficiently small, $u_{\epsilon}$ blows up at a boundary point that maximizes the mean curvature of $\partial \Omega$.

Since the publication of [38], problem (1.1) has received a great deal of attention and significant progress has been made. It has been proved that higher energy solutions exist, which concentrates at one or several points of

1991 Mathematics Subject Classification. 35J25, 35J20, 35B33, 35B40.

Key words and phrases. Triple Junctions, Singularly Perturbed Problems, Finite dimensional reduction . 
the boundary, or at one or more points in the interior, or a combination of the two effects. See [5], [4], [11]-[8], [20]-[17], [23]-[24], [39], [45]-[46]-[26] and the references therein. In particular, Lin, Ni and Wei [26] showed that there are at least $\frac{C_{N}}{(\epsilon|\log \epsilon|)^{N}}$ number of interior spikes.

It seems natural to ask if problem (1.1) has solutions which "concentrate" on higher dimensional sets, e.g. curves, or surfaces. In this regards, we mention that it has been conjectured for a long time that problem (1.1) actually possesses solutions which have $m$-dimensional concentration sets for every $0 \leq m \leq N-1$. (See e.g. [36].) Progress in this direction, although still limited, has also been made in $[2,30,31,32,33]$. In particular, we mention the results of Malchiodi and Montenegro [31, 32] on the existence of solutions concentrating on the whole boundary provided that the sequence $\varepsilon$ satisfies some gap condition. The latter condition is called resonance.

In the papers [27]-[30]-[32], the higher dimensional concentration set is on the boundary. A natural question is whether there are solutions with higher dimensional concentration set inside the domain. In this regard, the first result was due to Wei and Yang [47] who proved the existence of layer on the line intersecting with the boundary of a two-dimensional domain orthogonally. In [47] the resonance condition is still required. This result was generalized in [3] to domains of dimensions higher than 2.

By rescaling and taking a limit in (1.1), we obtain the following nonlinear elliptic problem in the whole $\mathbb{R}^{N}$

$$
\Delta u-u+u^{p}=0, \quad u>0 \text { in } \mathbb{R}^{N} .
$$

Recently there are several interesting results on new entire solutions to

$$
\Delta u-u+u^{p}=0, \quad u>0 \text { in } \mathbb{R}^{2} .
$$

Dancer [7] first constructed solutions to (1.3) which decays in one direction and periodic in another direction. In [14], these periodic solutions are called Dancer's solutions. Using Dancer's solutions, del Pino-KowalczykPacard-Wei built solutions to (1.3) with even number of ends whose level sets are governed by the one-dimensional Toda system. On the other hand, in [28], Malchiodi constructed another new class of entire solutions to (1.3) by perturbing a configuration of infinitely many copies of the positive solution $w$ arranged along three rays meeting at a common point, where $w$ is the unique radially symmetric solution of

$$
\left\{\begin{array}{c}
\Delta w-w+w^{p}=0, u>0 \text { in } \mathbb{R}^{2} \\
w(0)=\max _{y \in \mathbb{R}^{2}} w(y), w \rightarrow 0 \text { at } \infty
\end{array}\right.
$$

Malchiodi's solutions are known as triple-junction solutions. The question we address in this paper is whether or not there corresponds to a tripe junction solutions to (1.1) in a bounded domain.

The answer is yes: indeed, we construct triple junction solutions for Problem (1.1) which obtained as perturbation of a large number of $w$ centered at points arranged along a proper triple-junction . 


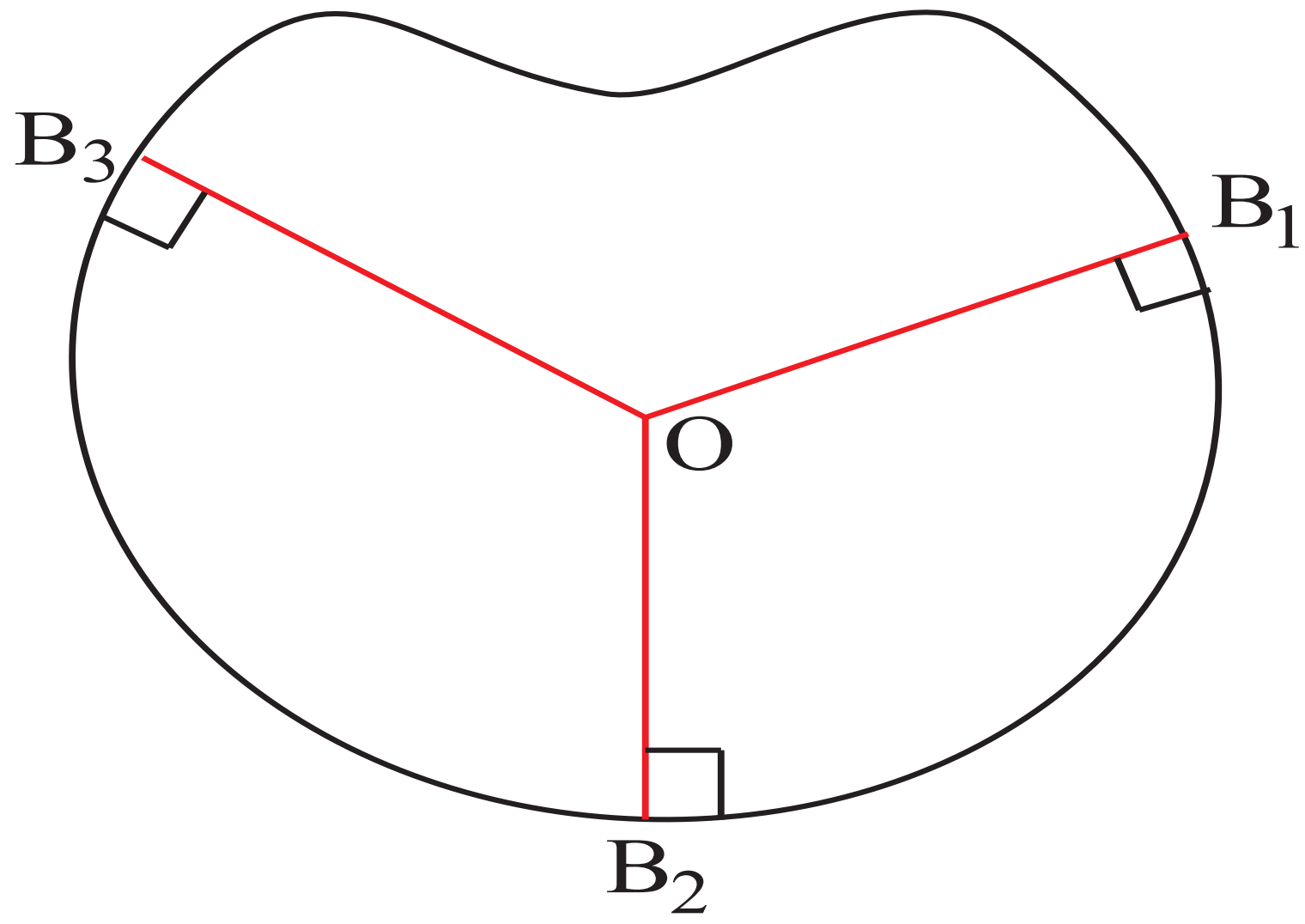

Figure 1. Triple Junctions

Let us first recall the asymptotic behavior of $w$, solution to (1.4), at infinity. It is known that there exists a constant $c_{p}>0$, depending on $p$, such that

$$
\lim _{r \rightarrow \infty} e^{r} r^{\frac{1}{2}} w=c_{p}>0, \quad \text { and } \quad \lim _{r \rightarrow \infty} \frac{w^{\prime}}{w}=-1,
$$

where we have set $r:=|x|$.

Furthermore, the solution $w$ is nondegenerate, namely the $L^{\infty}$-kernel of the operator

$$
L_{0}:=\Delta-1+p w^{p-1},
$$

which is nothing but the linearized operator about $w$, is spanned by the functions

$$
\partial_{x_{1}} w, \ldots, \partial_{x_{N}} w
$$

which naturally belong to this space. We refer the reader to ([37]).

Next we describe our result.

Assume that $\Omega$ contains three line segments with origin as the common endpoint which intersect orthogonally the boundary at exactly three points 
$B_{1}, B_{2}, B_{3}$ on $\partial \Omega$. (See Figure 1.) We denote by

$$
\mathbf{t}_{1}=\left(t_{11}, t_{12}\right), \quad \mathbf{t}_{2}=\left(t_{21}, t_{22}\right), \quad \mathbf{t}_{3}=(0,-1)
$$

the unit tangent vectors of the three segments, and by

$$
\mathbf{n}_{1}=\left(t_{12},-t_{11}\right), \quad \mathbf{n}_{2}=\left(t_{22},-t_{21}\right), \quad \mathbf{n}_{3}=(-1,0)
$$

respectively the unit normal vectors. Let $\overline{L_{1}}, \overline{L_{2}}, \overline{L_{3}}$ be the lengths of the three segments.

We assume that the mutual angles of the three lines satisfy that

$$
\mathbf{t}_{i} \angle \mathbf{t}_{j}>\frac{\pi}{3} \text {. }
$$

Near the endpoints $B_{1}, B_{2}$ and $B_{3}$ of the segments, the boundary $\partial \Omega$ is described as

$$
x \mathbf{n}_{1}+\left(h_{1}(x)+\overline{L_{1}}\right) \mathbf{t}_{1}, \quad x \mathbf{n}_{2}+\left(h_{2}(x)+\overline{L_{2}}\right) \mathbf{t}_{2}, \quad x \mathbf{n}_{3}+\left(h_{3}(x)+\overline{L_{3}}\right) \mathbf{t}_{3}
$$

respectively, where the functions $h_{i}$ are smooth functions defined in intervals which include 0 . It is not restrictive to assume that they satisfy $h_{i}(0)=$ $h_{i}^{\prime}(0)=0$ for all $i=1,2,3$.

Finally, we denote by $k_{i}$ the scalar curvature of $\partial \Omega$ at the point $B_{i}$, namely $k_{i}=-h_{i}^{\prime \prime}(0)$.

To state our result we need to introduce a function which, as we will show later, measures the interaction between two bumps $w$ centered at two distinct point. We define $\Psi(s)$ to be

$$
\Psi(s):=\int_{\mathbb{R}^{2}} w(x-s \mathbf{e}) \operatorname{div}\left(w^{p} \mathbf{e}\right) d x
$$

where $\mathbf{e}$ is a unit vector.

Our result is the following:

Theorem 1.1. Assume $\Omega \in \mathbb{R}^{2}$ contains three segments $\Gamma_{1}, \Gamma_{2}, \Gamma_{3}$ with origin as the common endpoint which intersects orthogonally the boundary of $\Omega$ in exactly three points $B_{1}, B_{2}$ and $B_{3}$ and whose lengths are $\bar{L}_{1}, \bar{L}_{2}$ and $\bar{L}_{3}$ respectively, and satisfy (1.10).

Assume that at least one $k_{i} \neq 1 / \bar{L}_{i}$.

Then there exist $\varepsilon_{0}>0$ such that, for any $0<\varepsilon<\varepsilon_{0}$ and for any real number $L_{3}$ such that

$$
c|\ln \varepsilon| \leq L_{3}, \quad \lim _{\varepsilon \rightarrow 0} \varepsilon L_{3}=0,
$$

for some positive constant $c>0$ which depends on $\Omega$ and on the lengths of the segments, if there exist positive real numbers $L_{1}, L_{2}$ and integers $m, n, l$ satisfying the following balancing formula

$$
\Psi\left(L_{1}\right) \boldsymbol{t}_{1}+\Psi\left(L_{2}\right) \boldsymbol{t}_{2}+\Psi\left(L_{3}\right) \boldsymbol{t}_{3}=\boldsymbol{O},
$$

and

$$
\left(m+\frac{1}{2}\right) L_{1}=\frac{\bar{L}_{1}}{\varepsilon},\left(n+\frac{1}{2}\right) L_{2}=\frac{\bar{L}_{2}}{\varepsilon},\left(l+\frac{1}{2}\right) L_{3}=\frac{\bar{L}_{3}}{\varepsilon},
$$


then there exists a solution $u_{\varepsilon}$ to Problem (1.1). Furthermore there exist $m+n+l$ points

$$
P_{j}^{\varepsilon}, \quad \text { for } \quad j=1, \ldots, m, \quad Q_{j}^{\varepsilon}, \quad \text { for } \quad j=1, \ldots, n,
$$

and

$$
R_{j}^{\varepsilon}, \quad \text { for } j=1, \ldots, l
$$

distributed uniformly at distance $\varepsilon L_{1}, \varepsilon L_{2}, \varepsilon L_{3}$ respectively along the segments $\Gamma_{1}, \Gamma_{2}, \Gamma_{3}$ and a point $O^{\varepsilon}$ near the origin such that

$u_{\varepsilon}(x)=\sum_{i=1}^{m} \sum_{j=1}^{n} \sum_{k=1}^{l}\left[w\left(\frac{x-P_{i}^{\varepsilon}}{\varepsilon}\right)+w\left(\frac{x-Q_{j}^{\varepsilon}}{\varepsilon}\right)+w\left(\frac{x-R_{k}^{\varepsilon}}{\varepsilon}\right)\right]+w\left(\frac{x-O^{\varepsilon}}{\varepsilon}\right)+o(1)$,

where $o(1) \rightarrow 0$ as $\varepsilon \rightarrow 0$ uniformly over compacts of $\mathbb{R}^{2}$.

Remark 1.1. As a consequence of the balancing condition (1.13) and the choice (1.8)-(1.9), without loss of generality we can assume that the mutual angles of the three lines satisfy that $\boldsymbol{t}_{1} \angle \boldsymbol{t}_{3} \geq \frac{\pi}{2}+\theta_{0}, \boldsymbol{t}_{2} \angle \boldsymbol{t}_{3} \geq \frac{\pi}{2}+\theta_{0}$ where $\theta_{0}$ is a constant. Thus we can get that $t_{i j} \neq 0$ for $i, j=1,2$.

Conditions (1.13)-(1.14) are satisfied under some restrictions on $\bar{L}_{i}$ or $\epsilon$. See the remarks below.

Remark 1.2. Let $L=L_{3}>>1$ be given and multiply relation (1.12) against $\boldsymbol{t}_{3}$ first and then against $\boldsymbol{n}_{3}$. This gives the system

$$
\begin{gathered}
\Psi\left(L_{1}\right) \boldsymbol{t}_{1} \cdot \boldsymbol{t}_{3}+\Psi\left(L_{2}\right) \boldsymbol{t}_{2} \cdot \boldsymbol{t}_{3}=-\Psi(L) \\
\Psi\left(L_{1}\right) \boldsymbol{t}_{1} \cdot \boldsymbol{n}_{3}+\Psi\left(L_{2}\right) \boldsymbol{t}_{2} \cdot \boldsymbol{n}_{3}=0
\end{gathered}
$$

This system is solvable since

$$
d=\left(\boldsymbol{t}_{1} \cdot \boldsymbol{t}_{3}\right)\left(\boldsymbol{t}_{2} \cdot \boldsymbol{n}_{3}\right)-\left(\boldsymbol{t}_{2} \cdot \boldsymbol{t}_{3}\right)\left(\boldsymbol{t}_{1} \cdot \boldsymbol{n}_{3}\right)=t_{12} t_{21}-t_{11} t_{22} \neq 0 .
$$

In this case, we thus have

$$
\Psi\left(L_{1}\right)=\frac{t_{21}}{d} \Psi(L), \quad \Psi\left(L_{2}\right)=-\frac{t_{11}}{d} \Psi(L) .
$$

Now, since $\Psi(s)=C_{p} e^{-s} s^{-\frac{1}{2}}\left(1+O\left(s^{-1}\right)\right)$ as $s \rightarrow \infty$, with $C_{p}$ a positive constant, we obtain that

$$
L_{1}=L-C_{1}, L_{2}=L-C_{2}
$$

where

$$
C_{1}=-\log \frac{t_{21}}{d}+O\left(\frac{1}{L}\right), C_{2}=-\log \left(-\frac{t_{11}}{d}\right)+O\left(\frac{1}{L}\right) .
$$

Then the condition (1.14) becomes

$$
\frac{\bar{L}_{3}}{\bar{L}_{1}}-\frac{2 l+1}{2 m+1}=\frac{\epsilon C_{1}(2 l+1)}{\bar{L}_{1}}, \frac{\bar{L}_{3}}{\bar{L}_{2}}-\frac{2 l+1}{2 n+1}=\frac{\epsilon C_{2}(2 l+1)}{\bar{L}_{2}}
$$


Remark 1.3. Let us consider the case $C_{1}=C_{2}=0$, i.e.,

$$
\boldsymbol{t}_{i} \angle \boldsymbol{t}_{j}=\frac{2 \pi}{3}
$$

In this case, condition (1.18) is satisfied if the ratios $\frac{\bar{L}_{3}}{L_{1}}, \frac{\bar{L}_{3}}{L_{2}}$ are of rational numbers of the form $\frac{2 r+1}{2 q+1}$. In this case, we let $\frac{\bar{L}_{3}}{\bar{L}_{1}}=\frac{2 r_{1}+1}{2 q_{1}+1}, \frac{\bar{L}_{3}}{\bar{L}_{2}}=\frac{2 r_{2}+1}{2 q_{2}+1}$. Then we choose $2 l+1=\left(2 r_{1}+1\right)\left(2 r_{2}+1\right)(2 k+1), 2 m+1=\left(2 q_{1}+1\right)\left(2 r_{2}+1\right)(2 k+$ $1), 2 n+1=\left(2 q_{2}+1\right)\left(2 r_{1}+1\right)(2 k+1)$, where $1<<k<\frac{C}{\varepsilon|\lg \varepsilon|}$. Then (1.12)(1.14) are satisfied.

Remark 1.4. We consider another case, $C_{1}=C_{2} \neq 0$, i.e., $L_{1}=L_{2} \neq L_{3}$. (We may assume that $C_{1}=C_{2}>0$.) In this case, we assume that $\bar{L}_{1}=\bar{L}_{2}$. Then we choose $m=n$ and $(m, l)$ such that

$$
\frac{\bar{L}_{3}}{\bar{L}_{1}}-\frac{2 l+1}{2 m+1}=-\frac{\epsilon C_{1}(2 l+1)}{\bar{L}_{1}}
$$

which is possible by the following choices: we may always choose a sequence of integers $m, l \rightarrow+\infty$ such that $\frac{\bar{L}_{3}}{\bar{L}_{1}}-\frac{2 l+1}{2 m+1} \sim-\frac{1}{m}$. Then (1.20) is satisfied if we choose a sequence $\epsilon_{(m, l)} \sim \frac{1}{m^{2}} \rightarrow$ satisfying (1.18).

If $C_{1} \neq C_{2}$, condition (1.18) is more complicated. Some conditions on the ratio $\frac{C_{1}}{C_{2}}$ are needed.

By the above remark, we now have the following corollary

Corollary 1.1. Assume $\Omega \in \mathbb{R}^{2}$ contains three segments $\Gamma_{1}, \Gamma_{2}, \Gamma_{3}$ with origin as the common endpoint which intersects orthogonally the boundary of $\Omega$ in exactly three points $B_{1}, B_{2}$ and $B_{3}$ and whose lengths are $\overline{L_{1}}, \overline{L_{2}}$ and $\bar{L}_{3}$ respectively, and satisfy (1.10). Assume that at least one $k_{i} \neq 1 / \bar{L}_{i}$. Furthermore

$$
\boldsymbol{t}_{i} \angle \boldsymbol{t}_{j}=\frac{2 \pi}{3}
$$

and the ratios $\frac{\bar{L}_{i}}{L_{j}}$ are rational numbers of the form $\frac{2 r+1}{2 q+1}$. Then problem (1.1) has a triple-junction solutions for $\epsilon$ sufficiently small.

Triple-junctions have appeared in many phase transition problems. In general they appear in vector-valued mimimization problems. See Sternberg [40] and Sternberg-Zimmer [41]. Bronsard-Gui-Schatzman [6] constructed symmetric layered solutions for the vectoral Allen-Cahn equation

$$
\Delta U-\nabla W(U)=0, U: \mathbb{R}^{2} \rightarrow \mathbb{R}^{2}
$$

and Gui-Schatzman [18] generalized to symmetric duadruple layered solutions. See also Alama-Bronsard-Gui [1]. As far as we know, Theorem 1.1 and Corollary 1.1 are the first results on the construction of triple-junctions in bounded domains. We believe that solutions concentrating on more complex networks of graphs may also exist. 
Acknowledgments. The research of the second author has been partly supported by Fondecyt Grant 1080099, Chile and CAPDE-Anillo ACT-125. The research of the third author is supported by a General Research Grant from RGC of Hong Kong.

\section{Ansatz And SKetch of the PROOF}

By the scaling $x=\varepsilon z$, problem (1.1) becomes

$$
\Delta u-u+u^{p}=0, \quad u>0 \quad \text { in } \quad \Omega_{\varepsilon}, \quad \frac{\partial u}{\partial \nu}=0 \quad \text { on } \quad \partial \Omega_{\varepsilon},
$$

where $\Omega_{\varepsilon}=\left\{\frac{x}{\varepsilon}: x \in \Omega\right\}$.

We consider a large number $L$, and $L_{1}, L_{2}, L_{3} \in \mathbb{R}$ for which the following condition hold

$$
\left|L_{i}-L\right| \leq C_{0}
$$

for $i=1,2,3$, where $C_{0}$ is a positive constant.

Define

$$
\left\{\begin{array}{c}
O=(\alpha, \beta) \\
P_{i}=\left(L_{1} i+a_{i}\right) \mathbf{t}_{1}+L_{1} b_{i} \mathbf{n}_{1}, \\
Q_{j}=\left(L_{2} j+c_{j}\right) \mathbf{t}_{2}+L_{2} d_{j} \mathbf{n}_{2}, \\
R_{k}=\left(L_{3} k+e_{k}\right) \mathbf{t}_{3}+L_{3} f_{k} \mathbf{n}_{3},
\end{array}\right.
$$

for $i=1, \cdots, m, j=1, \cdots, n, k=1, \cdots, l$, where the vector $\mathbf{t}_{i}$ and $\mathbf{n}_{i}$, $i=1,2,3$ are defined in (1.8) and (1.9).

We will assume that all the $\alpha, \beta, a_{i}, b_{i}, c_{j}, d_{j}, e_{k}, f_{k}$ are uniformly bounded, as $\varepsilon \rightarrow 0$. It will be convenient to adopt the following notations:

$$
\begin{aligned}
& \mathbf{a}=\left(a_{1}, a_{2}, \cdots, a_{m}\right), \mathbf{b}=\left(b_{1}, b_{2}, \cdots, b_{m}\right) \\
& \mathbf{c}=\left(c_{1}, c_{2}, \cdots, c_{n}\right), \mathbf{d}=\left(d_{1}, d_{2}, \cdots, d_{n}\right) \\
& \mathbf{e}=\left(e_{1}, e_{2}, \cdots, e_{l}\right), \mathbf{f}=\left(f_{1}, f_{2}, \cdots, f_{l}\right)
\end{aligned}
$$

We thus assume

$$
\|\mathbf{a}, \mathbf{b}, \mathbf{c}, \mathbf{d}, \mathbf{e}, \mathbf{f}, \alpha, \beta\| \leq c
$$

for some fixed $c>0$.

We will denote by $Y$ the set of all points, namely

$$
Y=\left\{z: z=O, P_{i}, Q_{j}, R_{k}, i=1, \cdots, m, j=1, \cdots, n, k=1, \cdots, l\right\} .
$$

Let us define the function

$$
U(x)=\sum_{z \in Y} U_{z}(x), \text { with } U_{z}(x)=w_{z}(x)-\varphi_{z}(x),
$$

where

$$
w_{z}(x)=w(x-z),
$$

and

$$
-\Delta \varphi_{z}+\varphi_{z}=0 \quad \text { in } \quad \Omega_{\varepsilon}, \quad \frac{\partial \varphi_{z}}{\partial \nu}=\frac{\partial w(x-z)}{\partial \nu} \quad \text { on } \quad \partial \Omega_{\varepsilon} .
$$

Next Lemma, whose proof is contained in [26], provides a qualitative description of the function $\varphi_{z}$. 
Lemma 2.1. Assume that $M|\ln \varepsilon| \leq d\left(P, \partial \Omega_{\varepsilon}\right) \leq \frac{\delta}{\varepsilon}$, for some constant $M$ depending on $N$ and a constant $\delta>0$ sufficiently small. Then

$$
\varphi_{P}(x)=-(1+o(1)) w\left(x-P^{*}\right)+o\left(\varepsilon^{3}\right),
$$

where $P^{*}=P+2 d\left(P, \partial \Omega_{\varepsilon}\right) \nu_{\bar{P}}, \nu_{\bar{P}}$ denotes the unit normal at $\bar{P}$ on $\partial \Omega_{\varepsilon}$, and $\bar{P}$ is the unique point on $\partial \Omega_{\varepsilon}$ such that $d(P, \bar{P})=d\left(P, \partial \Omega_{\varepsilon}\right)$.

We look for a solution of (2.1) of the form $u=U+\phi$. We set

$$
\begin{gathered}
L(\phi)=-\Delta \phi+\phi-p U^{p-1} \phi, \\
E=U^{p}-\sum_{z \in Y} w_{z}^{p},
\end{gathered}
$$

and

$$
N(\phi)=(U+\phi)^{p}-U^{p}-p U^{p-1} \phi .
$$

Problem (2.1) gets rewritten as

$$
L(\phi)=E+N(\phi) \quad \text { in } \quad \Omega_{\varepsilon}, \quad \frac{\partial \phi}{\partial \nu}=0 \quad \text { on } \quad \partial \Omega_{\varepsilon}
$$

Consider a cut off function $\chi \in C^{\infty}(0, \infty)$ such that

$$
\chi(s) \equiv 1 \quad \text { for } \quad s \leq-1, \quad \text { and } \quad \chi(s) \equiv 0 \quad \text { for } \quad s \geq 0 .
$$

We fix a constant $\zeta>0$ (independent of $L$ ) so that the balls of radius $\frac{L-\zeta}{2}$, centered at different points of $Y$ are mutually disjoint, for all $L$ large enough. We define the compactly supported functions

$$
Z_{z}(x):=\chi(2|x-z|-L+\zeta) \nabla w(x-z)
$$

for $z \in Y$. Observe that, by construction (in fact given the choice of $\zeta$ ), we have

$$
\int_{\Omega_{\varepsilon}} e_{i} \cdot Z_{z_{1}}(x) e_{j} \cdot Z_{z_{2}}(x) d x=0
$$

if $z_{1} \neq z_{2}$ or $i \neq j$.

Consider the following intermediate non linear projected problem: given the points in (2.3), find a function $\phi$ in some proper space and constant vectors $c_{z}$ such that

$$
\left\{\begin{array}{l}
L(\phi)=E+N(\phi)+\sum_{z \in Y} c_{z} Z_{z} \text { in } \Omega_{\varepsilon}, \\
\frac{\partial \phi}{\partial \nu}=0 \text { on } \partial \Omega_{\varepsilon}, \\
\int_{\Omega_{\varepsilon}} \phi Z_{z}=0 \text { for } z \in Y .
\end{array}\right.
$$

We show unique solvability of Problem (2.14) by means of a fixed point argument. Furthermore we prove that the solution $\phi$ depends smoothly on the points $z$. 
To do so, in Section 3 we develop a solvability theory for the linear projected problem

$$
\left\{\begin{array}{l}
L \phi=h+\sum_{z \in Y} c_{z} Z_{z} \text { in } \Omega_{\varepsilon}, \\
\frac{\partial \phi}{\partial \nu}=0 \text { on } \partial \Omega_{\varepsilon}, \\
\int_{\Omega_{\varepsilon}} \phi Z_{z}=0 \text { for } z \in Y,
\end{array}\right.
$$

for a given right hand side $h$ in some proper space. Roughly speaking, the linear operator $L$ is a super position of the linear operators

$$
L_{j} \phi=\Delta \phi-\phi+p w^{p-1}(x-z) \phi, \quad z \in Y .
$$

Once we have the unique solvability of Problem (2.14), which is proved in Section 4, it is clear that $u=U+\phi$ is indeed an exact solution to our original Problem (1.1), with the qualitative properties described in Theorem 1.1, if we can prove that the constants $c_{z}$ appearing in (2.14) are all zero. This can be done adjusting properly the parameters a,b,c,d,e,f, $\alpha, \beta$, as will be shown in Section 5, where the proof of Theorem 1.1 will be also given.

\section{LINEAR THEORY}

Our main result in this section states bounded solvability of Problem (2.15), uniformly in small $\varepsilon$, in points $z$ belonging to $Y$ given by (2.5), uniformly separated from each other at distance $O(L)$. Indeed we assume that the points $z$ given by (2.3) satisfy constraints (2.4).

Given $0<\eta<1$, consider the norms

$$
\|h\|_{*}=\sup _{x \in \Omega_{\varepsilon}}\left|\sum_{z} e^{\eta|x-z|} h(x)\right|,
$$

where $z \in Y$ with $Y$ defined in (2.5).

Proposition 3.1. Let $c>0$ be fixed. There exist positive numbers $\eta \in(0,1)$, $\varepsilon_{0}$ and $C$, such that for all $0 \leq \varepsilon \leq \varepsilon_{0}$, for all integer $m, n, l$ and positive real number $L_{i}$ given by (1.13) and satisfying (1.14), for any points $z, z \in Y$ in (2.5) given by (2.3) and satisfying (2.4), there is a unique solution $\left(\phi, c_{z}\right)$ to problem (2.15). Furthermore

$$
\|\phi\|_{*} \leq C\|h\|_{*} .
$$

The proof of the above Proposition, which we postpone to the end of this section, is based on Fredholm alternative Theorem for compact operator and an a-priori bound for solution to (2.15) that we state (and prove) next.

Proposition 3.2. Let $c>0$ be fixed. Let $h$ with $\|h\|_{*}$ bounded and assume that $\left(\phi, c_{z}\right)$ is a solution to (2.15). Then there exist positive numbers $\varepsilon_{0}$ and $C$, such that for all $0 \leq \varepsilon \leq \varepsilon_{0}$, for all integers $m, n, l$ and positive real numbers $L_{1}, L_{2}, L_{3}$ given by (1.13) and satisfying (1.14), for any points $z$, $z \in Y$ given by (2.3) and satisfying (2.4), one has

$$
\|\phi\|_{*} \leq C\|h\|_{*} .
$$


Proof. We argue by contradiction. Assume there exist $\phi$ solution to (2.15) and

$$
\|h\|_{*} \rightarrow 0, \quad\|\phi\|_{*}=1
$$

We prove that

$$
c_{z} \rightarrow 0 \text {. }
$$

Multiply the equation in (2.15) against any of the components of the function $Z_{z}$ defined in (2.12), that, with abuse of notation, we still denote by $Z_{z}$, and integrate in $\Omega_{\varepsilon}$, we get

$$
\int_{\Omega_{\varepsilon}} L \phi Z_{z}(x)=\int_{\Omega_{\varepsilon}} h Z_{z}+c_{z} \int_{\Omega_{\varepsilon}} Z_{z}^{2}
$$

since (2.13) holds true. Given the exponential decay at infinity of $\partial_{x_{i}} w$ and the definition of $Z_{z}$ in $(2.12)$, we get

$$
\int_{\Omega_{\varepsilon}} Z_{z}^{2}=\int_{\mathbb{R}^{N}}(\nabla w)^{2}+O\left(e^{-\delta L}\right) \quad \text { as } \quad L \rightarrow \infty
$$

for some $\delta>0$. On the other hand

$$
\left|\int_{\Omega_{\varepsilon}} h Z_{z}\right| \leq C\|h\|_{*} \int_{\Omega_{\varepsilon}} \nabla w(x-z) e^{-\eta|x-z|} \leq C\|h\|_{*} .
$$

Here and in what follows, $C$ stands for a positive constant independent of $\varepsilon$, as $\varepsilon \rightarrow 0$ (or equivalently independent of $L$ as $L \rightarrow \infty$ ). Finally, if we write $\tilde{Z}_{z}(x)=\nabla w(x-z)$ and $\chi=\chi(2|x-z|-L+\zeta)$, we have

$$
\begin{aligned}
-\int_{\Omega_{\varepsilon}} L \phi Z_{z}(x) & =\int_{B\left(z, \frac{L-\zeta}{2}\right)}\left[\Delta \tilde{Z}_{z}-\tilde{Z}_{z}+p w^{p-1}(x-z) \tilde{Z}_{z}\right] \chi \phi \\
& +\int_{\partial B\left(z, \frac{L-\zeta}{2}\right)} \phi \nabla\left(\chi(2|x-z|-L+\zeta) \tilde{Z}_{z}\right) \cdot \mathrm{n} \\
& -\int_{B\left(z, \frac{L-\zeta}{2}\right)} \phi\left(\tilde{Z}_{z} \Delta \chi+2 \nabla \chi Z_{z}\right) \\
& +p \int_{B\left(P_{j}, \frac{L-\zeta}{2}\right)}\left(U^{p-1}-w^{p-1}(x-z)\right) \phi \tilde{Z}_{z} \chi
\end{aligned}
$$

Next we estimate all the terms of the previous formula.

Since

$$
\Delta \tilde{Z}_{z}-\tilde{Z}_{z}+p w^{p-1}\left(x-P_{j}\right) \tilde{Z}_{z}=0
$$

we get the first term is 0 . Furthermore, using the estimates in (1.5), we have

$$
\begin{aligned}
& \left|\int_{\partial B\left(z, \frac{L-\zeta}{2}\right)} \phi \nabla\left(\chi(2|x-z|-L+\zeta) \tilde{Z}_{z}\right) \cdot \mathrm{n}\right| \\
& \leq C\|\phi\|_{*} \int_{\partial B\left(z j, \frac{L-\zeta}{2}\right)} e^{-(1+\eta)|x-z|}|x-z|^{-\frac{1}{2}} d x \\
& \leq C e^{-(1+\xi) \frac{L}{2}}\|\phi\|_{*}
\end{aligned}
$$


for some proper $\xi>0$. Using again (1.5), the third integral can be estimated as follows

$$
\begin{gathered}
\left|\int_{B\left(z, \frac{L-\zeta}{2}\right)} \phi\left(\tilde{Z}_{z} \Delta \chi(2|x-z|-L+\zeta)+2 \nabla \chi(2|x-z|-L+\zeta) \nabla \tilde{Z}_{z}\right)\right| \\
\leq C\|\phi\|_{*} \int_{\frac{L-\zeta}{2}-1}^{\frac{L-\zeta}{2}} e^{-(1+\eta) s} s^{\frac{1}{2}} d s \leq C e^{-(1+\xi) \frac{L}{2}}\|\phi\|_{*},
\end{gathered}
$$

again for some $\xi>0$. Finally, we observe that in $B\left(z, \frac{L-\zeta}{2}\right)$ that

$$
\left|U^{p-1}(x)-w^{p-1}(x-z)\right| \leq w^{p-2}(x-z)\left[\sum_{x_{i} \neq z} w\left(x-x_{i}\right)\right] .
$$

Having this, we conclude that

$$
\begin{gathered}
\left|p \int_{B\left(z, \frac{L-\zeta}{2}\right)}\left(U^{p-1}(x)-w^{p-1}(x-z)\right) \phi \tilde{Z}_{z} \chi(2|x-z|-L+\zeta)\right| \\
\leq C e^{-(1+\xi) \frac{L}{2}}\|\phi\|_{*}
\end{gathered}
$$

for a proper $\xi>0$, depending on $N$ and $p$. We thus conclude that

$$
\left|c_{z}\right| \leq C\left[e^{-(1+\xi) \frac{L}{2}}\|\phi\|_{*}+\|h\|_{*}\right] .
$$

Thus we get the validity of (3.4), since we are assuming $\|\phi\|_{*}=1$ and $\|h\|_{*} \rightarrow 0$.

Let now $\eta \in(0,1)$. It is easy to check that the function

$$
W:=\sum_{z \in Y} e^{-\eta|\cdot-z|}
$$

satisfies

$$
L W \leq \frac{1}{2}\left(\eta^{2}-1\right) W
$$

in $\Omega_{\varepsilon} \backslash \cup_{z \in Y} B(z, \rho)$ provided $\rho$ is fixed large enough (independently of $L$ ). Hence the function $W$ can be used as a barrier to prove the pointwise estimate

$$
|\phi|(x) \leq C\left(\|L \phi\|_{*}+\sum_{z}\|\phi\|_{L^{\infty}(\partial B(z, \rho))}\right) W(x),
$$

for all $x \in \Omega_{\varepsilon} \backslash \cup_{z \in Y} B(z, \rho)$.

Granted these preliminary estimates, the proof of the result goes by contradiction. Let us assume there exist a sequence of $L$ tending to $\infty$ and a sequence of solutions of (2.15) for which the inequality is not true. The problem being linear, we can reduce to the case where we have a sequence $L^{(n)}$ tending to $\infty$ and sequences $h^{(n)}, \phi^{(n)}, c^{(n)}$ such that

$$
\left\|h^{(n)}\right\|_{*} \rightarrow 0, \quad \text { and } \quad\left\|\phi^{(n)}\right\|_{*}=1
$$


But (3.4) implies that we also have

$$
\left|c^{(n)}\right| \rightarrow 0 .
$$

Then (3.8) implies that there exists $z^{(n)} \in Y$ (see (2.5) for the definition of $Y)$ such that

$$
\left\|\phi^{(n)}\right\|_{L^{\infty}\left(B\left(z^{(n)}, \rho\right)\right)} \geq C,
$$

for some fixed constant $C>0$. Using elliptic estimates together with AscoliArzela's theorem, we can find a sequence $z^{(n)}$ and we can extract, from the sequence $\phi^{(n)}\left(\cdot-z^{(n)}\right)$ a subsequence which will converge (on compact) to $\phi_{\infty}$ a solution of

$$
\left(\Delta-1+p w^{p-1}\right) \phi_{\infty}=0,
$$

in $\mathbb{R}^{2}$, which is bounded by a constant times $e^{-\eta|x|}$, with $\eta>0$. Moreover, recall that $\phi^{(n)}$ satisfies the orthogonality conditions in (2.15). Therefore, the limit function $\phi_{\infty}$ also satisfies

$$
\int_{\mathbb{R}^{2}} \phi_{\infty} \nabla w d x=0
$$

But the solution $w$ being non degenerate, this implies that $\phi_{\infty} \equiv 0$, which is certainly in contradiction with (3.9) which implies that $\phi_{\infty}$ is not identically equal to 0.

Having reached a contradiction, this completes the proof of the Proposition.

We can now prove Proposition 3.1.

Proof of Proposition 3.1. Consider the space

$$
\mathcal{H}=\left\{u \in H^{1}\left(\Omega_{\varepsilon}\right): \int_{\Omega_{\varepsilon}} u Z_{z}=0, \quad z \in Y\right\}
$$

Notice that the problem $(2.15)$ in $\phi$ gets re-written as

$$
\phi+K(\phi)=\bar{h} \quad \text { in } \mathcal{H},
$$

where $\bar{h}$ is defined by duality and $K: \mathcal{H} \rightarrow \mathcal{H}$ is a linear compact operator. Using Fredholm's alternative, showing that equation (3.10) has a unique solution for each $\bar{h}$ is equivalent to showing that the equation has a unique solution for $\bar{h}=0$, which in turn follows from Proposition 3.2. The estimate (3.2) follows directly from Proposition 3.2. This concludes the proof of Proposition (3.1).

In the following, if $\phi$ is the unique solution given by Proposition 3.1, we set

$$
\phi=\mathcal{A}(h) .
$$

Estimate (3.2) implies

$$
\|\mathcal{A}(h)\|_{*} \leq C\|h\|_{*} .
$$




\section{THE NON LINEAR PROJECTED PROBLEM}

For small $\varepsilon$, large $L$, and fixed points $z \in Y(2.5)$ given by (2.3) satisfying constraints (2.4) we show solvability in $\phi, c_{z}$ of the non linear projected problem

$$
\left\{\begin{array}{l}
L(\phi)=E+N(\phi)+\sum_{z \in Y} c_{z} Z_{z} \text { in } \Omega_{\varepsilon}, \\
\frac{\partial \phi}{\partial \nu}=0 \text { on } \partial \Omega_{\varepsilon}, \\
\int_{\Omega_{\varepsilon}} \phi Z_{z}=0 \text { for } z \in Y .
\end{array}\right.
$$

We have the validity of the following result

Proposition 4.1. Let $c>0$ be fixed. There exist positive numbers $\varepsilon_{0}, C$, and $\xi>0$ such that for all $\varepsilon \leq \varepsilon_{0}$, for all integers $m, n, l$ and positive real numbers $L_{i}$ given by 1.13 and satisfying (1.14), for any points $z, z \in$ $Y$ given by (2.3) and satisfying (2.4), there is a unique solution $\left(\phi, c_{z}\right)$ to problem (2.14). This solution depends continuously on the parameters of the construction (namely $\boldsymbol{a}, \boldsymbol{b}, \boldsymbol{c}, \boldsymbol{d}, \boldsymbol{e}, \boldsymbol{f}, \alpha, \beta$ ) and furthermore

$$
\|\phi\|_{*} \leq C e^{-\frac{(1+\xi)}{2} L} .
$$

Proof. The proof relies on the contraction mapping theorem in the $\|\cdot\|_{*}$-norm above introduced. Observe that $\phi$ solves (2.14) if and only if

$$
\phi=\mathcal{A}(E+N(\phi))
$$

where $\mathcal{A}$ is the operator introduced in (3.11). In other words, $\phi$ solves (2.14) if and only if $\phi$ is a fixed point for the operator

$$
T(\phi):=\mathcal{A}(E+N(\phi)) .
$$

Given $r>0$, define

$$
\mathcal{B}=\left\{\phi \in C^{2}\left(\Omega_{\varepsilon}\right):\|\phi\|_{*} \leq r e^{-\frac{(1+\xi)}{2} L}, \int_{\Omega_{\varepsilon}} \phi Z_{z}=0\right\} .
$$

We will prove that $T$ is a contraction mapping from $\mathcal{B}$ in itself.

To do so, we claim that

$$
\|E\|_{*} \leq C e^{-\frac{(1+\xi)}{2} L},
$$

and

$$
\|N(\phi)\|_{*} \leq C\left[\|\phi\|_{*}^{2}+\|\phi\|_{*}^{p}\right],
$$

for some fixed function $C$ independent of $L$, as $L \rightarrow \infty$. We postpone the proof of the estimates above to the end of the proof of this Proposition. Assuming the validity of (4.4) and (4.5) and taking into account (3.12), we have for any $\phi \in \mathcal{B}$

$$
\begin{aligned}
\|T(\phi)\|_{*} \leq & C\left[\|E+N(\phi)\|_{*}\right] \leq C\left[e^{-\frac{(1+\xi)}{2} L}+r^{2} e^{-(1+\xi) L}+r^{p} e^{-\frac{p(1+\xi)}{2} L}\right] \\
\leq & r e^{-\frac{(1+\xi)}{2} L}
\end{aligned}
$$


for a proper choice of $r$ in the definition of $\mathcal{B}$, since $p>1$.

Take now $\phi_{1}$ and $\phi_{2}$ in $\mathcal{B}$. Then it is straightforward to show that

$$
\begin{aligned}
\left\|T\left(\phi_{1}\right)-T\left(\phi_{2}\right)\right\|_{*} & \leq C\left\|N\left(\phi_{1}\right)-N\left(\phi_{2}\right)\right\|_{*} \\
& \leq C\left[\left\|\phi_{1}\right\|_{*}^{\min (1, p-1)}+\left\|\phi_{2}\right\|_{*}^{\min (1, p-1)}\right]\left\|\phi_{1}-\phi_{2}\right\|_{*} \\
& \leq o(1)\left\|\phi_{1}-\phi_{2}\right\|_{*} .
\end{aligned}
$$

This means that $T$ is a contraction mapping from $\mathcal{B}$ into itself.

To conclude the proof of this Proposition we are left to show the validity of (4.4) and (4.5). We start with (4.4).

Fix $z \in Y$ and consider the region $|x-z| \leq \frac{L}{2+\sigma}$, where $\sigma$ is a small positive number to be chosen later. In this region the error $E$, whose definition is in (2.9), can be estimated in the following way (see (1.5))

$$
\begin{aligned}
|E(x)| & \leq C\left[w^{p-1}(x-z) \sum_{x_{i} \neq z} w\left(x-x_{i}\right)+\sum_{x_{i} \neq z} w^{p}\left(x-x_{i}\right)\right] \\
& \leq C w^{p-1}(x-z) \sum_{x_{i} \neq z} e^{-\left(\frac{1}{2}+\frac{\sigma}{2(2+\sigma)}\right) L} \\
& \leq C w^{p-1}(x-z) e^{-\left(\frac{1}{2}+\frac{\sigma}{4(2+\sigma)}\right) L} e^{-\frac{\sigma}{4(2+\sigma)} L} \\
& \leq C w^{p-1}(x-z) e^{-\frac{1+\xi}{2} L},
\end{aligned}
$$

for a proper choice of $\xi>0$.

Consider now the region $|x-z|>\frac{L}{2+\sigma}$, for all $j$. Since $0<\mu<p-1$, we write $\mu=p-1-M$. From the definition of $E$, we get in the region under consideration

$$
\begin{aligned}
|E(x)| & \leq C\left[\sum_{z} w^{p}(x-z)\right] \leq C\left[\sum_{z} e^{-\mu|x-z|}\right] e^{-(p-\mu) \frac{L}{2+\sigma}} \\
& \leq\left[\sum_{z} e^{-\mu|x-z|}\right] e^{-\frac{1+M}{2+\sigma} L} \leq\left[\sum_{z} e^{-\mu|x-z|}\right] e^{-\frac{1+\xi}{2} L}
\end{aligned}
$$

for some $\xi>0$, if we chose $M$ and $\sigma$ small enough. From (4.6) and (4.7) we get (4.4).

We now prove (4.5). Let $\phi \in \mathcal{B}$. Then

$$
|N(\phi)| \leq\left|(U+\phi)^{p}-U^{p}-p U^{p-1} \phi\right| \leq C\left(\phi^{2}+|\phi|^{p}\right) .
$$

Thus we have

$$
\begin{aligned}
\left|\sum_{j} e^{\eta\left|x-P_{j}\right|} N(\phi)\right| & \leq C\|\phi\|_{*}\left(|\phi|+|\phi|^{p-1}\right) \\
& \leq C\left(\|\phi\|_{*}^{2}+\|\phi\|_{*}^{p}\right) .
\end{aligned}
$$


This gives (4.5).

A direct consequence of the fixed point characterization of $\phi$ given above together with the fact that the error term $E$ depends continuously (in the

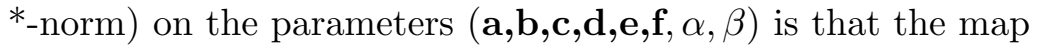

$$
((\mathrm{a}, \mathrm{b}, \mathrm{c}, \mathrm{d}, \mathrm{e}, \mathrm{f}), \alpha, \beta \rightarrow \phi
$$

into the space $C\left(\bar{\Omega}_{\varepsilon}\right)$ is continuous (in the $*$-norm). This concludes the proof of the Proposition.

Given points $z \in Y$, satisfying constraint (2.4), Proposition 4.1 guarantees the existence (and gives estimates) of a unique solution $\phi, c_{z}, z \in Y$, to Problem (2.14). It is clear then that the function $u=U+\phi$ is an exact solution to our problem (1.1), with the required properties stated in Theorem 1.1 if we show that there exists a configuration for the points $z$ that gives all the constants $c_{z}$ in (2.14) equal to zero. In order to do so we first need to find the correct conditions on the points to get $c_{z}=0$. This condition is naturally given by projecting in $L^{2}\left(\Omega_{\varepsilon}\right)$ the equation in (2.14) into the space spanned by $Z_{z}$, namely by multiplying the equation in (2.14) by $Z_{z}$ and integrate all over $\Omega_{\varepsilon}$. We will do it in details in the next final Section.

\section{ERror Estimates AND THE PROOF OF THEOREM 1.1}

The first aim of this section is to evaluate the $L^{2}\left(\Omega_{\varepsilon}\right)$ projection of the error term $E$ in (2.9) against the elements $Z_{z}$ in (2.12), for any $z \in Y$ in (2.5).

Let us introduce the following notations.

Let $P_{m}^{*}=P_{m}+2 d\left(P_{m}, \partial \Omega_{\varepsilon}\right) \nu_{\bar{P}_{m}}$, where $\nu_{\bar{P}_{m}}$ denotes the unit normal at $\bar{P}_{m}$ on $\partial \Omega_{\varepsilon}$ and $\bar{P}_{m}$ is the unique point on $\partial \Omega_{\varepsilon}$ such that $d\left(P_{m}, \bar{P}_{m}\right)=$ $d\left(P_{m}, \partial \Omega_{\varepsilon}\right)$. In analogous way we define $Q_{n}^{*}, \bar{Q}_{n}$ and $R_{l}^{*}, \bar{R}_{l}$.

Thus there exist three coordinates $x_{1}, x_{2}$ and $x_{3}$ such that

$\bar{P}_{m}=L_{1} x_{1} \mathbf{n}_{1}+\left(\frac{\bar{L}_{1}+h_{1}\left(\epsilon L_{1} x_{1}\right)}{\epsilon}\right) \mathbf{t}_{1}, \quad \bar{Q}_{n}=L_{2} x_{2} \mathbf{n}_{2}+\left(\frac{\bar{L}_{2}+h_{2}\left(\epsilon L_{2} x_{2}\right)}{\epsilon}\right) \mathbf{t}_{2}$,

and

$$
\bar{R}_{l}=L_{3} x_{3} \mathbf{n}_{3}+\left(\frac{\bar{L}_{3}+h_{3}\left(\epsilon L_{3} x_{3}\right)}{\epsilon}\right) \mathbf{t}_{3} .
$$

More explicitly, the coordinates $x_{i}$ are defined as solutions of the following system

$$
\left\{\begin{array}{c}
L_{1}\left(x_{1}-b_{m}\right)+\left(\frac{L_{1}}{2}+\frac{h_{1}\left(L_{1} \epsilon x_{1}\right)}{\epsilon}-a_{m}\right) h_{1}^{\prime}\left(L_{1} \epsilon x_{1}\right)=0 \\
L_{2}\left(x_{2}-d_{n}\right)+\left(\frac{L_{2}}{2}+\frac{h_{2}\left(L_{2} \epsilon x_{2}\right)}{h_{3} \epsilon}-c_{n}\right) h_{2}^{\prime}\left(L_{2} \epsilon x_{2}\right)=0 \\
L_{3}\left(x_{3}-f_{l}\right)+\left(\frac{L_{3}}{2}+\frac{h_{3}\left(L_{3} \epsilon x_{3}\right)}{\epsilon}-e_{l}\right) h_{3}^{\prime}\left(L_{3} \epsilon x_{3}\right)=0
\end{array}\right.
$$

We have the validity of the following 
Lemma 5.1. Let us define

$$
\kappa_{i}=-(\log \Psi)^{\prime}\left(L_{i}\right) .
$$

The following expansions hold true

$$
\begin{gathered}
\int_{w_{\varepsilon}} E Z_{P_{i}} d x=-\Psi\left(L_{1}\right)\left[\kappa_{1}\left(a_{i+1}-2 a_{i}+a_{i-1}\right) \boldsymbol{t}_{1}-\left(b_{i+1}-2 b_{i}+b_{i-1}\right) \boldsymbol{n}_{1}\right] \\
+e^{-\delta L_{1}} A+\Psi\left(L_{1}\right) Q
\end{gathered}
$$

for $i=2, \cdots, m-1$.

$$
\begin{gathered}
\int_{w_{\varepsilon}} E Z_{Q_{j}} d x=-\Psi\left(L_{2}\right)\left[\kappa_{2}\left(c_{j+1}-2 c_{j}+c_{j-1}\right) \boldsymbol{t}_{2}-\left(d_{j+1}-2 d_{j}+d_{j-1}\right) \boldsymbol{n}_{2}\right] \\
+e^{-\delta L_{2}} A+\Psi\left(L_{2}\right) Q
\end{gathered}
$$

for $j=2, \cdots, n-1$.

$$
\begin{gathered}
\int_{\Omega_{\varepsilon}} E Z_{R_{k}} d x=-\Psi\left(L_{3}\right)\left[\kappa_{3}\left(e_{k+1}-2 e_{k}+e_{k-1}\right) \boldsymbol{t}_{3}-\left(f_{k+1}-2 f_{k}+f_{k-1}\right) \boldsymbol{n}_{3}\right] \\
+e^{-\delta L_{3}} A+\Psi\left(L_{3}\right) Q
\end{gathered}
$$

for $k=2, \cdots, l-1$.

$$
\begin{gathered}
\int_{w_{\varepsilon}} E Z_{P_{1}} d x=-\Psi\left(L_{1}\right)\left[\kappa_{1}\left(a_{2}-2 a_{1}+(\alpha, \beta) \boldsymbol{t}_{1}\right) \boldsymbol{t}_{1}-\left(b_{2}-2 b_{1}+\frac{(\alpha, \beta) \boldsymbol{n}_{1}}{L_{1}} \boldsymbol{n}_{1}\right)\right] \\
+e^{-\delta L_{1}} A+\Psi\left(L_{1}\right) Q \\
\int_{w_{\varepsilon}} E Z_{Q_{1}} d x=-\Psi\left(L_{2}\right)\left[\kappa_{2}\left(c_{2}-2 c_{1}+(\alpha, \beta) \boldsymbol{t}_{2}\right) \boldsymbol{t}_{2}-\left(d_{2}-2 d_{1}+\frac{(\alpha, \beta) \boldsymbol{n}_{2}}{L_{2}} \boldsymbol{n}_{2}\right)\right] \\
+e^{-\delta L_{2}} A+\Psi\left(L_{2}\right) Q \\
\int_{w_{\varepsilon}} E Z_{R_{1}} d x=-\Psi\left(L_{3}\right)\left[\kappa_{3}\left(e_{2}-2 e_{1}+(\alpha, \beta) \boldsymbol{t}_{3}\right) \boldsymbol{t}_{3}-\left(f_{2}-2 f_{1}+\frac{(\alpha, \beta) \boldsymbol{n}_{3}}{L_{3}} \boldsymbol{n}_{3}\right)\right] \\
+e^{-\delta L_{3}} A+\Psi\left(L_{3}\right) Q, \\
\int_{w_{\epsilon}} E Z_{P_{m}} d x=-\Psi\left(L_{1}\right)\left[\kappa_{1}\left(a_{m-1}-3 a_{m}+\frac{2 h_{1}\left(L_{1} \varepsilon x_{1}\right)}{\varepsilon}\right) \boldsymbol{t}_{1}-\left(b_{m-1}-3 b_{m}+2 x_{1}\right) \boldsymbol{n}_{1}\right] \\
+e^{-\delta L_{1}} A+\Psi\left(L_{1}\right) Q, \\
\left.\int_{w_{\epsilon}} E Z_{Q_{n}} d x=-\Psi\left(L_{2}\right)\left[\kappa_{2}\left(c_{n-1}-3 c_{n}+\frac{2 h_{2}\left(L_{2} \varepsilon x_{2}\right)}{\varepsilon}\right) \boldsymbol{t}_{2}-\left(d_{n-1}-3 d_{n}+2 x_{2}\right) \boldsymbol{n}_{2}\right)\right] \\
+e^{-\delta L_{2}} A+\Psi\left(L_{2}\right) Q, \\
\int_{w_{\epsilon}} E Z_{R_{l}} d x=-\Psi\left(L_{3}\right)\left[\kappa_{3}\left(e_{l-1}-3 e_{l}+\frac{2 h_{3}\left(L_{3} \varepsilon x_{3}\right)}{\varepsilon}\right) \boldsymbol{t}_{3}-\left(f_{l-1}-3 f_{l}+2 x_{3}\right) \boldsymbol{n}_{3}\right] \\
+e^{-\delta L_{3}} A+\Psi\left(L_{3}\right) Q
\end{gathered}
$$




$$
\begin{gathered}
\int_{\Omega_{\varepsilon}} E Z_{O} d x=\Psi\left(L_{1}\right)\left[\kappa_{1}\left(a_{1}-(\alpha, \beta) \boldsymbol{t}_{1}\right) \boldsymbol{t}_{1}+\left(b_{1}-\frac{(\alpha, \beta) \boldsymbol{n}_{1}}{L_{1}}\right) \boldsymbol{n}_{1}\right] \\
+\Psi\left(L_{2}\right)\left[\kappa_{2}\left(c_{1}-(\alpha, \beta) \boldsymbol{t}_{2}\right) \boldsymbol{t}_{2}+\left(d_{1}-\frac{(\alpha, \beta) \boldsymbol{n}_{2}}{L_{2}}\right) \boldsymbol{n}_{2}\right] \\
+\Psi\left(L_{3}\right)\left[\kappa_{3}\left(e_{1}-(\alpha, \beta) \boldsymbol{t}_{3}\right) \boldsymbol{t}_{3}+\left(f_{1}-\frac{(\alpha, \beta) \boldsymbol{n}_{3}}{L_{3}}\right) \boldsymbol{n}_{3}\right] \\
+e^{-\delta L} A+\Psi(L) Q
\end{gathered}
$$

Furthermore,

$$
\int L(\phi) Z_{z}=e^{-\delta L} A, \quad z \in Y
$$

and

$$
\int N(\phi) Z_{z}=e^{-\delta L} A \quad z \in Y
$$

where $\delta>1, A=A(\boldsymbol{a}, \boldsymbol{b}, \boldsymbol{c}, \boldsymbol{d}, \boldsymbol{e}, \boldsymbol{f}, \boldsymbol{x}, \alpha, \beta)$ and $Q=Q(\boldsymbol{a}, \boldsymbol{b}, \boldsymbol{c}, \boldsymbol{d}, \boldsymbol{e}, \boldsymbol{f}, \boldsymbol{x}, \alpha, \beta) d e-$ note the smooth vector valued functions (which vary from line to line), uniformly bounded as $L \rightarrow \infty$ and the Taylor expansion of $Q$ with respect to $\boldsymbol{a}, \boldsymbol{b}, \boldsymbol{c}, \boldsymbol{d}, \boldsymbol{e}, \boldsymbol{f}, \boldsymbol{x}, \alpha, \beta$ does not involve any constant nor any linear terms.

Proof. Observe that, given $\mathrm{e} \in \mathbb{R}^{2}$ with $|\mathrm{e}|=1$ and $\mathrm{a} \in \mathbb{R}^{N}$, a direct consequence of estimates (1.5) is that the following expansion holds

$$
\Psi(|\tilde{L} \mathrm{e}+\mathrm{a}|) \frac{(\tilde{L} \mathrm{e}+\mathrm{a})}{|\tilde{L} \mathrm{e}+\mathrm{a}|}=\Psi(\tilde{L})\left(\mathrm{e}-\tilde{\kappa} \mathrm{a}^{\|}+\frac{1}{\tilde{L}} \mathrm{a}^{\perp}+O\left(|\mathrm{a}|^{2}\right)\right)
$$

as $\tilde{L} \rightarrow \infty$, where $\tilde{\kappa}=-(\log \Psi)^{\prime}(\tilde{L})$. Here, we have decomposed a $=\mathrm{a}^{\| l}+\mathrm{a}^{\perp}$ where $\mathrm{a}^{\|}$is collinear to e and $\mathrm{a}^{\perp}$ is orthogonal to e. See also [34].

Estimates (5.2)-(5.4) are by now standard, see for instance [34]. For completeness, we show

$$
\begin{aligned}
\int_{w_{\varepsilon}} E Z_{P_{i}}(x) d x & =\int_{R^{2}} w\left(x-P_{i-1}\right) p w^{p-1}\left(x-P_{i}\right) \nabla w\left(x-P_{i}\right) d x \\
& +\int_{R^{2}} w\left(x-P_{i+1}\right) p w^{p-1}\left(x-P_{i}\right) \nabla w\left(x-P_{i}\right) d x+e^{-\delta L_{1}} A \\
& =-\Psi\left(L_{1}\right)\left(\kappa_{1}\left(a_{i+1}-2 a_{i}+a_{i-1}\right) \mathbf{t}_{1}-\left(b_{i+1}-2 b_{i}+b_{i-1}\right) \mathbf{n}_{1}\right) \\
& +e^{-\delta L_{1}} A+\Psi\left(L_{1}\right) Q,
\end{aligned}
$$

for $i=2, \cdots, m-1$.

Similarly we can get the two equations for $Q_{j}, R_{k}$.

Concerning estimates (5.5)-(5.7), a direct use of (5.14) gives

$$
\begin{aligned}
\int_{\Omega_{\varepsilon}} E Z_{P_{1}} d x & =\int_{R^{2}} w(x-O) p w^{p-1}\left(x-P_{1}\right) \nabla w\left(x-P_{1}\right) d x \\
& +\int_{R^{2}} w\left(x-P_{2}\right) p w^{p-1}\left(x-P_{1}\right) \nabla w\left(x-P_{1}\right) d x+e^{-\delta L_{1}} A \\
& =-\Psi\left(L_{1}\right)\left(\kappa_{1}\left(a_{2}-2 a_{1}+(\alpha, \beta) \mathbf{t}_{1}\right) \mathbf{t}_{1}-\left(b_{2}-2 b_{1}\right) \mathbf{n}_{1}-\frac{(\alpha, \beta) \mathbf{n}_{1}}{L_{1}} \mathbf{n}_{1}\right) \\
& +e^{-\delta L_{2}} A+\Psi\left(L_{1}\right) Q .
\end{aligned}
$$


Similarly we can get the two equations for $Q_{1}, R_{1}$.

To compute (5.8)-(5.10), we use the result of Lemma 2.1. Given (5.1) we obtain that

$$
\left\{\begin{array}{c}
P_{m}^{*}-P_{m}=2\left(x_{1}-b_{m}\right) L_{1} \mathbf{n}_{1}+\left(L_{1}+\frac{2 h_{1}\left(\epsilon L_{1} x_{1}\right)}{\epsilon}-2 a_{m}\right) \mathbf{t}_{1} \\
Q_{n}^{*}-Q_{n}=2\left(x_{2}-d_{n}\right) L_{2} \mathbf{n}_{2}+\left(L_{2}+\frac{2 h_{2}\left(\epsilon L_{2} x_{2}\right)}{\epsilon}-2 c_{n}\right) \mathbf{t}_{2}, \\
R_{l}^{*}-R_{l}=2\left(x_{3}-f_{l}\right) L_{3} \mathbf{n}_{3}+\left(L_{3}+\frac{2 h_{3}\left(\epsilon L_{3} x_{3}\right)}{\epsilon}-2 e_{l}\right) \mathbf{t}_{3} .
\end{array}\right.
$$

Thus a direct use of Lemma 2.1 gives estimate (5.8) as follows

$$
\begin{aligned}
\int_{w_{\epsilon}} E Z_{P_{m}} d x & =\int_{\Omega_{\varepsilon}} p w^{p-1}\left(x-P_{m}\right) \nabla w\left(x-P_{m}\right) w\left(x-P_{m-1}\right) \\
& +\int_{\Omega_{\varepsilon}} p w^{p-1}\left(x-P_{m}\right) \nabla w\left(x-P_{m}\right) w\left(x-P_{m}^{*}\right)+e^{-\delta L_{1}} A \\
& =-\Psi\left(L_{1}\right)\left(\kappa_{1}\left(3 a_{m}-a_{m-1}-\frac{2 h_{1}\left(L_{1} \epsilon x_{1}\right)}{\epsilon}\right) \mathbf{t}_{1}\right. \\
& \left.+\left(2 x_{1}+b_{m-1}-3 b_{m}\right) \mathbf{n}_{1}\right)+e^{-\delta L_{1}} A+\Psi\left(L_{1}\right) Q
\end{aligned}
$$

In the same way we get the equations for $Q_{n}, R_{l}$.

Finally, expansion (5.11) is given by

$$
\begin{aligned}
\int_{\Omega_{\varepsilon}} E Z_{O}(x) d x & =\int_{\Omega_{\varepsilon}} p w^{p-1}(x-O) w\left(x-P_{1}\right) \nabla w(x-O) d x \\
& +\int_{w_{\epsilon}} p w^{p-1}(x-O) w\left(x-Q_{1}\right) \nabla w(x-O) d x \\
& +\int_{w_{\epsilon}} p w^{p-1}(x-O) w\left(x-R_{1}\right) \nabla w(x-O) d x+e^{-\delta L} A \\
& =-\Psi\left(L_{1}\right)\left(\mathbf{t}_{1}-\kappa_{1}\left(a_{1}-(\alpha, \beta) \mathbf{t}_{1}\right) \mathbf{t}_{1}+\frac{L_{1} b_{1}-(\alpha, \beta) \mathbf{n}_{1}}{L_{1}} \mathbf{n}_{1}\right) \\
& -\Psi\left(L_{2}\right)\left(\mathbf{t}_{2}-\kappa_{2}\left(c_{1}-(\alpha, \beta) \mathbf{t}_{2}\right) \mathbf{t}_{2}+\frac{L_{2} d_{1}-(\alpha, \beta) \mathbf{n}_{2}}{L_{2}} \mathbf{n}_{2}\right) \\
& -\Psi\left(L_{3}\right)\left(\mathbf{t}_{3}-\kappa_{3}\left(e_{1}-(\alpha, \beta) \mathbf{t}_{3}\right) \mathbf{t}_{3}+\frac{L_{3} f_{1}-(\alpha, \beta) \mathbf{n}_{3}}{L_{3}} \mathbf{n}_{3}\right) \\
& +e^{-\delta L} A+Q \Psi(L) .
\end{aligned}
$$

The proof of (5.12) follows the line of the proof of Proposition 3.2 (see formula (3.6) and the subsequent estimates, together with (4.2)).

The proof of (5.13) follows from estimate (4.5) and (4.2). 
For any integer $k$ let us now define the following $k \times k$ matrix

$$
T:=\left(\begin{array}{ccccc}
2 & -1 & 0 & \ldots & 0 \\
-1 & \ddots & \ddots & \ddots & \vdots \\
0 & \ddots & \ddots & \ddots & 0 \\
\vdots & \ddots & \ddots & 2 & -1 \\
0 & \ldots & 0 & -1 & 2
\end{array}\right) \in \mathcal{M}_{k \times k}
$$

It is well known that the matrix $T$ is invertible and its inverse is the matrix whose entries are given by

$$
\left(T^{-1}\right)_{i j}=\min (i, j)-\frac{i j}{k+1} .
$$

We define the vectors $S^{\downarrow}$ and $S^{\uparrow}$ by

$$
T S^{\downarrow}:=\left(\begin{array}{c}
0 \\
\vdots \\
0 \\
1
\end{array}\right) \in \mathbb{R}^{k}, \quad T S^{\uparrow}:=\left(\begin{array}{c}
1 \\
0 \\
\vdots \\
0
\end{array}\right) \in \mathbb{R}^{k}
$$

It is immediate to check that

$$
S^{\downarrow}:=\left(\begin{array}{c}
\frac{1}{k+1} \\
\frac{2}{k+1} \\
\vdots \\
\frac{k-1}{k+1} \\
\frac{k}{k+1}
\end{array}\right) \in \mathbb{R}^{k}, \quad S^{\uparrow}:=\left(\begin{array}{c}
\frac{k}{k+1} \\
\frac{k-1}{k+1} \\
\vdots \\
\frac{2}{k+1} \\
\frac{1}{k+1}
\end{array}\right) \in \mathbb{R}^{k} .
$$

With this in mind we have that the above lemma gives the validity of the following

Lemma 5.2. The coefficients $c_{z}$ in Problem (4.1) are all equal to 0 if and only if the parameters $\boldsymbol{a}, \boldsymbol{b}, \boldsymbol{c}, \boldsymbol{d}, \boldsymbol{e}, \boldsymbol{f}, \alpha, \beta$ are solutions of the nonlinear system

$$
\left\{\begin{array}{c}
\boldsymbol{a}=\left(\frac{2 h_{1}\left(\varepsilon L_{1} x_{1}\right)}{\epsilon}-a_{m}\right) S^{\downarrow}+(\alpha, \beta) \cdot \boldsymbol{t}_{1} S^{\uparrow}+e^{-\delta L} A+Q \in \mathbb{R}^{m}, \\
\boldsymbol{b}=\left(2 x_{1}-b_{m}\right) S^{\downarrow}+\frac{(\alpha, \beta) \cdot n_{1}}{L_{1}} S^{\uparrow}+e^{-\delta L} A+Q \in \mathbb{R}^{m}, \\
\boldsymbol{c}=\left(\frac{2 h_{2}\left(\varepsilon L_{2} x_{2}\right)}{\epsilon}-c_{n}\right) S^{\downarrow}+(\alpha, \beta) \cdot \boldsymbol{t}_{2} S^{\uparrow}+e^{-\delta L} A+Q \in \mathbb{R}^{2}, \\
\boldsymbol{d}=\left(2 x_{2}-d_{n}\right) S^{\downarrow}+\frac{(\alpha, \beta) \cdot \boldsymbol{n}_{2}}{L_{2}} S^{\uparrow}+e^{-\delta L} A+Q \in \mathbb{R}^{2}, \\
\boldsymbol{e}=\left(\frac{2 h_{3}\left(\varepsilon L_{3} x_{3}\right)}{\epsilon}-e_{l}\right) S^{\downarrow}+(\alpha, \beta) \cdot \boldsymbol{t}_{3} S^{\uparrow}+e^{-\delta L} A+Q \in \mathbb{R}^{l}, \\
\boldsymbol{f}=\left(2 x_{3}-f_{l}\right) S^{\downarrow}+\frac{(\alpha, \beta) \cdot \boldsymbol{n}_{3}}{L_{3}} S^{\uparrow}+e^{-\delta L} A+Q \in \mathbb{R}^{l},
\end{array}\right.
$$


where $\delta>0$ and $x_{1}, x_{2}$ and $x_{3}$ are given by (5.1). Furthermore $\alpha, \beta$ satisfy

$$
\begin{aligned}
& -\Psi\left(L_{1}\right)\left(-\kappa_{1}\left(a_{1}-(\alpha, \beta) \cdot \boldsymbol{t}_{1}\right) \boldsymbol{t}_{1}+\left(b_{1}-\frac{(\alpha, \beta) \cdot \boldsymbol{n}_{1}}{L_{1}}\right) \boldsymbol{n}_{1}\right) \\
& -\quad \Psi\left(L_{2}\right)\left(-\kappa_{2}\left(c_{1}-(\alpha, \beta) \cdot \boldsymbol{t}_{2}\right) \boldsymbol{t}_{2}+\left(d_{1}-\frac{(\alpha, \beta) \cdot \boldsymbol{n}_{2}}{L_{2}}\right) \boldsymbol{n}_{2}\right) \\
& -\quad \Psi\left(L_{3}\right)\left(-\kappa_{3}\left(e_{1}-(\alpha, \beta) \cdot \boldsymbol{t}_{3}\right) \boldsymbol{t}_{3}+\left(f_{1}-\frac{(\alpha, \beta) \cdot \boldsymbol{n}_{3}}{L_{3}}\right) \boldsymbol{n}_{3}\right) \\
& +\quad e^{-\delta_{1} L} A+Q \Psi(L)=0 .
\end{aligned}
$$

In this last formula $\delta_{1}>1$. In the above formula we have denoted by $A=A(\boldsymbol{a}, \boldsymbol{b}, \boldsymbol{c}, \boldsymbol{d}, \boldsymbol{e}, \boldsymbol{f}, \alpha, \beta)$ and $Q=Q(\boldsymbol{a}, \boldsymbol{b}, \boldsymbol{c}, \boldsymbol{d}, \boldsymbol{e}, \boldsymbol{f}, \alpha, \beta)$ smooth vector valued functions (which vary from line to line), uniformly bounded as $L \rightarrow \infty$ and the Taylor expansion of $Q$ with respect to $\boldsymbol{a}, \boldsymbol{b}, \boldsymbol{c}, \boldsymbol{d}, \boldsymbol{e}, \boldsymbol{f}, \alpha, \beta$ does not involve any constant nor any linear term.

Given the result of the above lemma, we are left to show that (5.19)-(5.20) have a solution $(\mathbf{a}, \mathbf{b}, \mathbf{c}, \mathbf{d}, \mathbf{e}, \mathbf{f}, \alpha, \beta)$ with

$$
\|(\mathbf{a}, \mathbf{b}, \mathbf{c}, \mathbf{d}, \mathbf{e}, \mathbf{f}, \alpha, \beta)\| \leq c
$$

for some positive $c$, small and independent of $\varepsilon$.

We first observe that using the assumptions that $h_{i}(0)=h_{i}^{\prime}(0)=0$ for all $i=1,2,3$, from the equations (5.1) satisfied by $x_{1}, x_{2}, x_{3}$, we get that

$$
\left\{\begin{array}{c}
x_{1}=\left(1-\frac{\bar{L}_{1} h_{1}^{\prime \prime}(0)}{2 m+1}\right) b_{m}+e^{-\delta L} A+Q, \\
x_{2}=\left(1-\frac{L_{2} h_{2}^{\prime \prime}(0)}{2 n+1}\right) d_{n}+e^{-\delta L} A+Q, \\
x_{3}=\left(1-\frac{\bar{L}_{3} h_{2}^{\prime \prime}(0)}{2 l+1}\right) f_{l}+e^{-\delta L} A+Q,
\end{array}\right.
$$

for some constant $\delta>0$.

On the other hand, using the expressions for $S^{\uparrow}$ and $S^{\downarrow}$ given by (5.18), from the first two equations in (5.19) get that

$$
\left\{\begin{array}{c}
a_{1}=\frac{2 h_{1}\left(\varepsilon L_{1} x_{1}\right)}{(2 m+1) \epsilon}+\frac{2 m-1}{2 m+1}(\alpha, \beta) \cdot \mathbf{t}_{1}+e^{-\delta L} A+Q, \\
b_{1}=\frac{2 x_{1}}{2 m+1}+\frac{2 m-1}{(2 m+1) L_{1}}(\alpha, \beta) \cdot \mathbf{n}_{1}+e^{-\delta L} A+Q, \\
a_{m}=\frac{2 m h_{1}\left(\varepsilon L_{1} x_{1}\right)}{(2 m+1) \epsilon}+\frac{1}{2 m+1}(\alpha, \beta) \cdot \mathbf{t}_{1}+e^{-\delta L} A+Q, \\
b_{m}=\frac{2 m x_{1}}{2 m+1}+\frac{1}{(2 m+1) L_{1}}(\alpha, \beta) \cdot \mathbf{n}_{1}+e^{-\delta L} A+Q .
\end{array}\right.
$$

In a very similar way from the last four equations in (5.19) we get the expressions of $c_{1}, c_{n}, d_{1}, d_{n}, e_{1}, e_{l}, f_{1}, f_{l}$.

Using (5.21) and (5.22), from (5.20) we can get that the parameters $\alpha, \beta$ satisfy the system

$$
\left\{\begin{array}{c}
B \alpha-C \beta=\frac{2 \Psi\left(L_{1}\right)}{2 m+1} t_{12} x_{1}+\frac{2 \Psi\left(L_{2}\right)}{2 n+1} t_{22} x_{2}-\frac{2 \Psi\left(L_{3}\right)}{2 k+1} x_{3}+e^{-\delta L} A+Q \Psi(L), \\
C \alpha-D \beta=\frac{2 \Psi\left(L_{1}\right)}{2 m+1} t_{11} x_{1}+\frac{2 \Psi\left(L_{2}\right)}{2 n+1} t_{21} x_{2}+e^{-\delta L} A+Q \Psi(L),
\end{array}\right.
$$


for some $\delta>1$, where the constants $B, C$ and $D$ are given by

$$
\begin{gathered}
B=\frac{2 \Psi\left(L_{1}\right)}{(2 m+1) L_{1}} t_{12}^{2}+\frac{2 \Psi\left(L_{2}\right)}{(2 n+1) L_{2}} t_{22}^{2}+\frac{2 \Psi\left(L_{3}\right)}{(2 k+1) L_{3}}-\frac{\Psi\left(L_{1}\right)}{2 m+1} t_{11}^{2}-\frac{\Psi\left(L_{2}\right)}{2 n+1} t_{21}^{2} \\
C=\frac{2 \Psi L_{1}}{(2 m+1) L_{1}} t_{11} t_{12}+\frac{2 \Psi\left(L_{2}\right)}{(2 n+1) L_{2}} t_{21} t_{22}+\frac{\Psi\left(L_{1}\right)}{2 m+1} t_{11} t_{12}+\frac{\Psi\left(L_{2}\right)}{2 n+1} t_{21} t_{22} \\
D=\frac{2 \Psi\left(L_{1}\right)}{(2 m+1) L_{1}} t_{11}^{2}+\frac{2 \Psi\left(L_{2}\right)}{(2 n+1) L_{2}} t_{21}^{2}-\frac{\Psi\left(L_{1}\right)}{2 m+1} t_{12}^{2}-\frac{\Psi\left(L_{2}\right)}{2 n+1} t_{22}^{2}-\frac{\Psi\left(L_{3}\right)}{2 k+1}
\end{gathered}
$$

Recall that the numbers $t_{i j}$ are the components of the vectors $\mathbf{t}_{1}$ and $\mathbf{t}_{2}$ in (1.8). A direct computation shows that the system in $\alpha$ and $\beta$ is uniquely solvable, since

$$
\begin{gathered}
C^{2}-B D=-\frac{\Psi\left(L_{1}\right) \Psi\left(L_{3}\right)}{(2 m+1)(2 l+1)} t_{11}^{2}-\frac{\Psi\left(L_{2}\right) \Psi\left(L_{3}\right)}{(2 n+1)(2 l+1)} t_{21}^{2} \\
-\frac{\Psi\left(L_{1}\right) \Psi\left(L_{2}\right)}{(2 m+1)(2 n+1)}\left(t_{11} t_{22}-t_{12} t_{21}\right)^{2} \neq 0,
\end{gathered}
$$

given the fact that we have already observed that it is not restrictive to assume that all $t_{i j} \neq 0$ for $i, j=1,2$.

One can check that

$$
\left\{\begin{array}{l}
\alpha=\frac{1}{C^{2}-B D}\left(C \frac{2 \Psi\left(L_{1}\right)}{2 m+1}\right) t_{11} x_{1}+C \frac{2 \Psi\left(L_{2}\right.}{2 n+1} t_{21} x_{2}-D \frac{2 \Psi\left(L_{1}\right)}{2 m+1} t_{12} x_{1}-D \frac{2 \Psi\left(L_{2}\right)}{2 n+1} t_{22} x_{2}+D \frac{2 \Psi\left(L_{3}\right)}{2 k+1} x_{3} \\
\beta=\frac{1}{C^{2}-B D}\left(B \frac{2 \Psi\left(L_{1}\right)}{2 m+1}\right) t_{11} x_{1}+B \frac{2 \Psi\left(L_{2}\right)}{2 n+1} t_{21} x_{2}-C \frac{2 \Psi\left(L_{1}\right)}{2 m+1} t_{12} x_{1}-C \frac{2 \Psi\left(L_{2}\right)}{2 n+1} t_{22} x_{2}+C \frac{2 \Psi\left(L_{3}\right)}{2 k+1} x_{3}
\end{array}\right.
$$

Replacing these values of $\alpha$ and $\beta$, together with (5.21), in equations (5.22) and in the corresponding equations for the parameters $c_{1}, c_{n}, d_{1}, d_{n}, e_{1}, e_{l}, f_{1}, f_{l}$, we obtain that the whole problem is reduced to the solvability of the following non linear system in the variables $b_{1}, b_{m}, d_{1}, d_{n}, f_{1}, f_{l}$

$$
\left(\begin{array}{ll}
T_{1} & T_{2} \\
T_{3} & T_{4}
\end{array}\right)\left(b_{1}, d_{1}, f_{1}, b_{m}, d_{n}, f_{l}\right)^{t}=e^{-\delta L} A+Q
$$

where

$$
\begin{aligned}
& T_{1}=-I_{3 \times 3}, \\
& T_{3}=O_{3 \times 3},
\end{aligned}
$$

and

$$
T_{4}=\left(\begin{array}{ccc}
1+\frac{2 m}{2 m+1} \bar{L}_{1} h_{1}^{\prime \prime}+A_{1} & A_{2} & A_{3} \\
B_{1} & 1+\frac{2 n}{2 n+1} \bar{L}_{2} h_{2}^{\prime \prime}+B_{2} & B_{3} \\
C_{1} & C_{2} & 1+\frac{2 l}{2 l+1} \bar{L}_{3} h_{3}^{\prime \prime}+C_{3}
\end{array}\right)
$$


with the constants $A_{j}, B_{j}$ and $C_{j}$ defined as follows

$$
\left\{\begin{array}{c}
A_{1}=\frac{2 \Psi\left(L_{1}\right)}{\left(C^{2}-B D\right)(2 m+1) L_{1}}\left(B t_{11}^{2}-2 C t_{11} t_{12}+D t_{12}^{2}\right)\left(1-\frac{\bar{L}_{1} h_{1}^{\prime \prime}(0)}{2 m+1}\right), \\
A_{2}=\frac{2 \Psi\left(L_{2}\right)}{\left(C^{2}-B D\right)(2 n+1) L_{1}}\left(B t_{21} t_{11}-C t_{11} t_{22}-C t_{21} t_{12}+D t_{12} t_{22}\right)\left(1-\frac{\bar{L}_{2} h_{2}^{\prime \prime}(0)}{2 n+1}\right), \\
A_{3}=\frac{2 \Psi\left(L_{3}\right)}{\left(C^{2}-B D\right)(2 k+1) L_{1}}\left(C t_{11}-D t_{12}\right)\left(1-\frac{\bar{L}_{3} h_{3}^{\prime \prime}(0)}{2 l+1}\right) \\
B_{1}=\frac{2 \Psi\left(L_{1}\right)}{\left(C^{2}-B D\right)(2 m+1) L_{2}}\left(B t_{11} t_{21}-C t_{12} t_{21}-C t_{11} t_{22}+D t_{12} t_{22}\right)\left(1-\frac{\bar{L}_{1} h_{1}^{\prime \prime}(0)}{2 m+1}\right) \\
B_{2}=\frac{2 \Psi\left(L_{2}\right)}{\left(C^{2}-B D\right)(2 n+1) L_{2}}\left(B t_{21}^{2}-2 C t_{21} t_{22}+D t_{22}^{2}\right)\left(1-\frac{\bar{L}_{2} h_{2}^{\prime \prime}(0)}{2 n+1}\right) \\
B_{3}=\frac{2 \Psi\left(L_{3}\right)}{\left(C^{2}-B D\right)(2 k+1) L_{2}}\left(C t_{21}-D t_{22}\right)\left(1-\frac{\bar{L}_{3} h_{3}^{\prime \prime}(0)}{2 l+1}\right), \\
C_{1}=\frac{2 \Psi\left(L_{1}\right)}{\left(C^{2}-B D\right)(2 m+1) L_{3}}\left(C t_{11}-D t_{12}\right)\left(1-\frac{\bar{L}_{1} h_{1}^{\prime \prime}(0)}{2 m+1}\right) \\
C_{2}=\frac{2 \Psi\left(L_{2}\right)}{\left(C^{2}-B D\right)(2 n+1) L_{3}}\left(C t_{21}-D t_{22}\right)\left(1-\frac{\bar{L}_{2} h_{2}^{\prime \prime}(0)}{2 n+1}\right) \\
C_{3}=\frac{2 \Psi\left(L_{3}\right)}{\left(C^{2}-B D\right)(2 k+1) L_{3}} D\left(1-\frac{\bar{L}_{3} h_{3}^{\prime \prime}(0)}{2 l+1}\right)
\end{array}\right.
$$

In order to solve (5.27), we need to compute the determinant of the matrix $\left(\begin{array}{ll}T_{1} & T_{2} \\ T_{3} & T_{4}\end{array}\right)$

Set $H_{i}=1+\bar{L}_{i} h_{i}^{\prime \prime}(0)$. We write

$$
\operatorname{det}\left(\begin{array}{ll}
T_{1} & T_{2} \\
T_{3} & T_{4}
\end{array}\right)=\sum_{j=1}^{4} \Delta_{j},
$$

where

$$
\begin{gathered}
\Delta_{1}=\left(H_{1}-\frac{\bar{L}_{1} h_{1}^{\prime \prime}(0)}{2 m+1}\right)\left(H_{2}-\frac{\bar{L}_{2} h_{2}^{\prime \prime}(0)}{2 n+1}\right)\left(H_{3}-\frac{\bar{L}_{3} h_{3}^{\prime \prime}(0)}{2 l+1}\right), \\
\Delta_{2}=A_{1}\left(H_{2}-\frac{\bar{L}_{2} h_{2}^{\prime \prime}(0)}{2 n+1}\right)\left(H_{3}-\frac{\bar{L}_{3} h_{3}^{\prime \prime}(0)}{2 l+1}\right)+B_{2}\left(H_{1}-\frac{\bar{L}_{1} h_{1}^{\prime \prime}(0)}{2 m+1}\right)\left(H_{3}-\frac{\bar{L}_{3} h_{3}^{\prime \prime}(0)}{2 l+1}\right) \\
+C_{3}\left(H_{1}-\frac{\bar{L}_{1} h_{1}^{\prime \prime}(0)}{2 m+1}\right)\left(H_{2}-\frac{\bar{L}_{2} h_{2}^{\prime \prime}(0)}{2 n+1}\right), \\
\Delta_{3}=\left(C_{3} B_{2}-C_{2} B_{3}\right)\left(H_{1}-\frac{\bar{L}_{1} h_{1}^{\prime \prime}(0)}{2 m+1}\right)+\left(A_{1} C_{3}-A_{3} C_{1}\right)\left(H_{2}-\frac{\bar{L}_{2} h_{2}^{\prime \prime}(0)}{2 n+1}\right) \\
+\left(A_{1} B_{2}-A_{2} B_{1}\right)\left(H_{3}-\frac{\bar{L}_{3} h_{3}^{\prime \prime}(0)}{2 l+1}\right)
\end{gathered}
$$

and

$$
\Delta_{4}=\operatorname{det}\left(\begin{array}{lll}
A_{1} & A_{2} & A_{3} \\
B_{1} & B_{2} & B_{3} \\
C_{1} & C_{2} & C_{3}
\end{array}\right) .
$$

Using the expression of the constants $A_{j}, B_{j}$ and $C_{j}$ in (5.28), an involved but direct computation gives that

$$
\Delta_{4}=0
$$


On the other hand, we observe the following: from the definition of the constant $A_{1}, B_{2}$ and $C_{3}$ in (5.28) and from the expression of $C^{2}-B D$ in (5.25), we get that

$$
A_{1}, B_{2}, C_{3}=M\left(\mathbf{t}_{i}, \bar{L}_{i}, C_{0}\right) \frac{1}{L},
$$

where $M$ is uniformly bounded from below away from zero as $\varepsilon \rightarrow 0$ (or equivalently as $L \rightarrow \infty$ ).

Furthermore, from the definition of

$$
A_{1} C_{3}-C_{1} A_{3}=\frac{\Psi\left(L_{1}\right) \Psi\left(L_{3}\right)}{(2 m+1)(2 l+1)\left(B D-C^{2}\right) L_{1} L_{3}} t_{11}^{2},
$$

we get that $\left|A_{1} C_{3}-C_{1} A_{3}\right| \geq c_{0} \frac{1}{L^{2}}$ as $\epsilon \rightarrow 0$ where $c_{0}>0$. In fact we get

$$
C_{3} B_{2}-B_{3} C_{2}, A_{1} C_{3}-C_{1} A_{3}, A_{1} B_{2}-B_{1} A_{2}=N\left(\mathbf{t}_{1}, \bar{L}_{i}, C_{0}\right) \frac{1}{L^{2}},
$$

where $N$ is uniformly bounded from below away from zero as $L \rightarrow \infty$.

We thus conclude that under the assumption that at least one $H_{i}$ is nonzero, the non linear system (5.27) can be uniquely solved by fixed point theorem of contraction mapping.

\section{REFERENCES}

[1] S. Alama, L. Bronsard and C. Gui, Stationary layerd solutions in $R^{2}$ for an AllenCahn system with mutiple well potential, Cal. Var. PDE 5(1997), 359-390.

[2] A. Ambrosetti, A. Malchiodi and W. M. Ni, Singularly perturbed elliptic equations with symmetry: existence of solutions concentrating on spheres, II, Indiana Univ. Math. J. 53 (2004), no. 2, 297-329.

[3] W. Ao, M. Musso and J. Wei, On Spikes concentrating on line-segments to a semilinear Neumann problem, preprint 2010.

[4] P. Bates, E. N. Dancer and J. Shi, Multi-spike stationary solutions of the CahnHilliard equation in higher-dimension and instability, Adv. Diff. Eqns 4 (1999), no. 1, 1-69.

[5] P. Bates and G. Fusco, Equilibria with many nuclei for the Cahn-Hilliard equation, J. Diff. Eqns. 160 (2000), no. 2, 283-356.

[6] L. Bronsard, C. Gui and M. Schatzman, A three-layered minimizer in $R^{2}$ for a variational problem with a symmetric three-well potential, Comm. Pure Appl. Math. 49(1996), 677-715.

[7] E.N. Dancer, New solutions of equations on $\mathbf{R}^{n}$, Ann. Scuola Norm. Sup. Pisa Cl. Sci. (4) 30 (2001), no. 3-4, 535-563.

[8] E. N. Dancer and S. Yan, Multipeak solutions for a singularly perturbed Neumann problem, Pacific J. Math. 189 (1999), no. 2, 241-262.

[9] E. N. Dancer and S. Yan, Interior and boundary peak solutions for a mixed boundary value problem, Indiana Univ. Math. J. 48 (1999), 1177-1212.

[10] T. D'Aprile and A. Pistoia, On the existence of some new positive interior spike solutions to a semilinear Neumann problem, J. Diff. Eqns. 248(2010), 556-573.

[11] M. del Pino, P. Felmer and J. Wei, On the role of mean curvature in some singularly perturbed Neumann problems, SIAM J. Math. Anal. 31 (1999), no. 1, 63-79.

[12] del Pino, M.; Felmer, P.; Wei, J.-C. Mutiple peak solutions for some singular perturbation problems. Cal. Var. PDE 10 (2000), 119-134.

[13] M. del Pino, P. Felmer and J. Wei, On the role of distance function in some singular perturbation problems, Comm. PDE 25 (2000), no. 1-2, 155-177. 
[14] M. del Pino, M. Kowalcyzk, F. Pacard, J. Wei, The Toda system and multiple-end solutions of autonomous planar elliptic problems. to appear in Advances in Math. 2010.

[15] A. Floer and A. Weinstein, Nonspreading wave packets for the cubic Schrödinger equation with bounded pontential, J. Func. Anal. 69 (1986), 397-408.

[16] A. Gierer and H. Meinhardt, A theory of biological pattern formation, Kybernetik(Berlin) 12 (1972), 30-39.

[17] M. Grossi, A. Pistoia and J. Wei, Existence of multipeak solutions for a semilinear Neumann problem via nonsmooth critical point theory, Calc. Var. PDE 11 (2000), no. 2, 143-175.

[18] C. Gui and M. Schatzman, Symmetric quadruple phase transitions, Indiana Univ. Math. J. 57(2008), no.2, 781-836.

[19] C. Gui and J. Wei, Multiple interior peak solutions for some singularly perturbed Neumann problems, J. Diff. Eqns. 158 (1999), no. 1, 1-27.

[20] C. Gui and J. Wei, On multiple mixed interior and boundary peak solutions for some singularly perturbed Neumann problems, Canad. J. Math. 52 (2000), no. 3, 522-538.

[21] C. Gui, J. Wei and M. Winter, Multiple boundary peak solutions for some singularly perturbed Neumann problems, Ann. Inst. H. Poincaré Anal. Non Linéaire 17 (2000), no. $1,47-82$

[22] Kwong, M.K. Uniquness of positive solutions of $\Delta u-u+u^{p}=0$ in $\mathbb{R}^{N}$. Arch. Rational Mech. Anal. 105 (1991), 243-266.

[23] Y.-Y. Li, On a singularly perturbed equation with Neumann boundary condition, Comm. PDE 23 (1998), no. 3-4, 487-545.

[24] Y.-Y. Li and L. Nirenberg, The Dirichlet problem for singularly perturbed elliptic equations, Comm. Pure Appl. Math. 51 (1998), no. 11-12, 1445-1490.

[25] C. S. Lin, W. M. Ni and I. Takagi, Large amplitude stationary solutions to a chemotaxis systems, J. Diff. Eqns. 72 (1988), no. 1, 1-27.

[26] F.H. Lin, W.M. Ni and J.C. Wei, On the number of interior p eak solutions for a singularly perturbed Neumann problem, Comm. Pure Appl. Math. 60 (2007), 252-281.

[27] F. Mahmoudi and A. Malchiodi, Concentration on minimal submanifolds for a sigularly pertubed Neumann problem, Advances in Mathematics 209 (2007), no. 2, 460-525.

[28] Malchiodi, A., Some new entire solutions of semilinear elliptic equations in $\mathbb{R}^{N}$, Adances in Math. 2009, to appear.

[29] A. Malchiodi, Solutions concentrating at curves for some singularly perturbed elliptic problems, C. R. Math. Acad. Sci. Parirs 338 (2004), no. 10, 775-780.

[30] A. Malchiodi, Concentration at curves for a singularly perturbed Neumann problem in three-dimensiional domains, Geom. Funct. Anal. 15 (2005), no. 6, 1162-1222.

[31] A. Malchiodi and M. Montenegro, Boundary concentration phenomena for a singularly perturbed elliptic problem, Comm. Pure Appl. Math, 55 (2002), no. 12, 15071568.

[32] A. Malchiodi and M. Montenegro, Multidimensional boundary layers for a singularly perturbed Neumann problem, Duke Math. J. 124 (2004), no. 1, 105-143.

[33] A. Malchiodi, W. M. Ni and J. Wei, Multiple clustered layer solutions for semilinear Neumann problems on a ball, Ann. Inst. H. Poincaré Anal. Non Linéaire 22 (2005), no. 2, 143-163.

[34] M. Musso, F. Pacard and J. Wei, Finite-energy sign-changing solutions with dihedral symmetry for the stationary non linear schrödinger equation, preprint 2009.

[35] W. M. Ni, Diffusion, cross-diffusion, and their spike-layer steady states, Notices of the AMS 45 (1998), no. 1, 9-18.

[36] W. M. Ni, Qualitative properties of solutions to elliptic problems, Stationary partial differential equations, Vol. I, 157-233, Handb. Differ. Equ., North-Holland, Amsterdam, 2004 
[37] W. M. Ni and I. Takagi, On the shape of least energy solution to a semilinear Neumann problem, Comm. Pure Appl. Math. 41 (1991), no. 7, 819-851.

[38] W. M. Ni and I. Takagi, Locating the peaks of least energy solutions to a semilinear Neumann problem, Duke Math. J. 70 (1993), no. 2, 247-281.

[39] W. M. Ni and J. Wei, On the location and profile of spike-layer solutions to singularly perturbed semilinear Dirichlet problems, Comm. Pure Appl. Math. 48 (1995), no. 7, 731-768.

[40] P. Sternberg, Vector-valued local minimizers of nonconvex variational problems, Rocky Mountain J. Math. 21(1991), 799-807.

[41] P. Sternberg and W. Zeimer, Local minimizers of a three-phase partition problem with triple junctions, Proc. Roy. Soc. Edinburgh Sect. A 124(1994), 1059-1073.

[42] J. Wei, On the boundary spike layer solutions to a singularly perturbed Neumann problem, J. Diff. Eqns. 134 (1997), no. 1, 104-133.

[43] J. Wei, On the interior spike layer solutions to a singularly perturbed Neumann problem, Tohoku Math. J. (2) 50 (1998), no. 2, 159-178.

[44] J. Wei, Existence and Stability of Spikes for the Gierer-Meinhardt System, Handbook of differential equations, stationary partial differential equadtions, volume 5 (M. Chipot ed.), Elservier. pp. 489-581.

[45] J. Wei and M. Winter, Stationary solutions for the Cahn-Hilliard equation, Ann. Inst. H. Poincaré Anal. Non Linéaire 15 (1998), no. 4, 459-492.

[46] J. Wei and M. Winter, Multi-peak solutions for a wide class of singular perturbation problems, J. London Math. Soc. 59 (1999), no. 2, 585-606.

[47] J. Wei and J. Yang, Concentration on lines for a singularly perturbed Neumann problem in two-dimensional domains, Indiana University Mathematics Journal, 56 (2007), no.6, 3025-3074.

W. Ao - Department of Mathematics, The Chinese University of Hong Kong, Shatin, Hong Kong.

E-mail address: wwao@math.cuhk.edu.hk

M. Musso - Departamento de Matemática, Pontificia Universidad Catolica de Chile, Avda. Vicuña Mackenna 4860, Macul, Chile

E-mail address: mmusso@math.upl.cl

J. Wei - Department of Mathematics, The Chinese University of Hong Kong, Shatin, Hong Kong.

E-mail address: wei@math.cuhk.edu.hk 